THE KURUME MEDICAL JOURNAL Vol.10, No.1, 1963

\title{
STUDY ON THE CORRELATION BETWEEN CLINICAL AND PHYSIOLOGICAL RESPONSES TO CHLORPROMAZINE
}

\author{
HIROSHI MUKASA AND KAZUTOYO INANAGA \\ Department of Neuropsychiatry, Kurume University School of Medicine, \\ Kurume-shi, Japan
}

(Received for publication January 31, 1963)

\begin{abstract}
Although chlorpromazine is one of the psychotherapeutic drugs used widely, its therapeutic effects have been inadequately correlated with its physiological response pattern. The authors and their co-workers ${ }^{1 / 3) 45) 911112)}$ have observed the effects of psychotherapeutic drugs on the EEG and minor tremor (MT) for the past several years, noticing that there exists a definite relation between the clinical and MT response pattern. The purpose of this article is to investigate whether a relation exists between the clinical and physiological responses to chlorpromazine, as indicated by EEG and MT.
\end{abstract}

\section{METHOD}

Subjects were selected at random from hospitalized schizophrenics in the University Hospital. They were 10 male and 2 female patients. The control recordings of EEG and MT before medication were done at a time more than a week after discontinuation of various drugs. The administration of chlorpromazine was started with $100 \mathrm{mg}$ per day for the first week and gradually increased by $100 \mathrm{mg}$ per day every week. The usual dosage was between 200 and $600 \mathrm{mg}$ per day. The recordings of EEG and MT were done before medication, one week after the administration of chlorpromazine and afterwards once a week for two or six weeks. Subjects were in the supine position and five minutes after lying down, EEG tracings were taken and thereafter MT was recorded. The EEG recorded from the leads between the right occipital and the right central area was analyzed by wave analyser (San'ei model EA-101). The MT on the left thenar was recorded and analysed. The duration of frequency analyses of EEG and MT was six epochs (60 minutes). The wave analyzer divides the 6-13 c/s frequency range of EEG and MT into seven frequency bands. The frequency curve was made by calculating the percentage of the squared integrated value of a given band to the sum of the squared integrated values of seven bands.

\section{RESULTS}

(1) EEG (Figs. 1 and 2)

The frequency curve of EEG before medication in each subject is seen in Figs. 1 


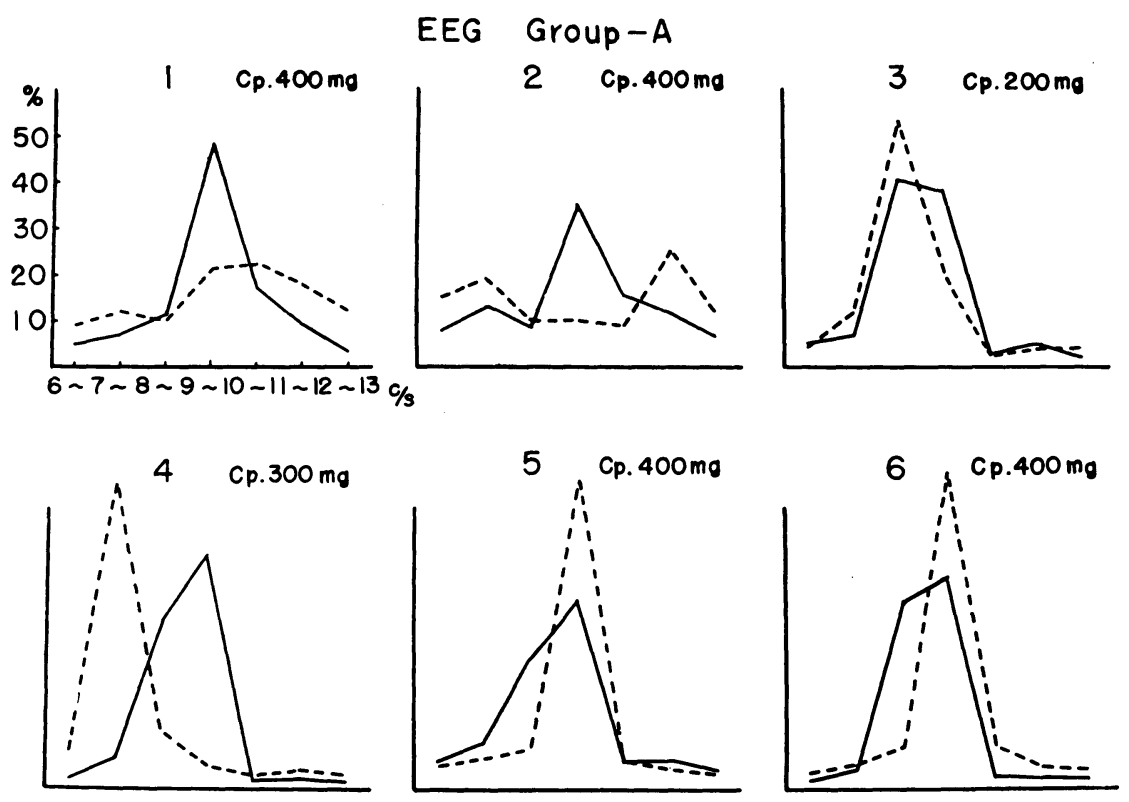

Fig. 1. Frequency graphs of EEG of 6 cases (Group A) before medication $(\cdots \cdots \cdots)$ and at the time of clinical improvement ( - ), during chlorpromazine therapy. The dosages per day of chlorpromazine $(\mathrm{Cp})$ are indicated.

Abscissa: frequency band

Ordinate: percentage of appearance of each frequency band

and 2, showing 6 cases with alpha dominant pattern, 4 cases without any dominant peak and 2 cases with the peak in the theta band. The frequency curves of six subjects, at the time when they were recognized as remarkably improved clinically by the doctors and nurses after the medication, were noticed to be evidently altered from those before medication (Fig. 1, Group-A). For example, the flat curve shifted to alpha-dominant (Cases 1 and 2), theta-dominant to alpha-dominant (Case 4) and extraordinary alpha-dominant shifted to normal (Cases 5 and 6). Representative frequency curves of six patients without improvement at any time during the chlorpromazine treatment (Fig. 2, Group-B) indicate minimum shifts from the curves before treatment. In cases 5 and $6,200 \mathrm{mg}$ per day of chlorpromazine did not change the frequency curves before medication at all and their clinical symptomes became worse, which made it impossible to continue further experimentation.

(2) MT (Figs 3 and 4)

Frequency curves of MT in all cases before medication show slight difference between Groups A and B. In Group A, four cases dominating at faster frequency bands, one case dominating at $9-10 \mathrm{c} / \mathrm{s}$, and one case dominating at $8-9 \mathrm{c} / \mathrm{s}$ were observed. In Group B, frequency curves dominating at the faster frequency bands were seen in all cases. After medication, the dominant peak was observed at 11-12 $\mathrm{c} / \mathrm{s}$ in all cases of Group A while there were diverse changes in Group B. 


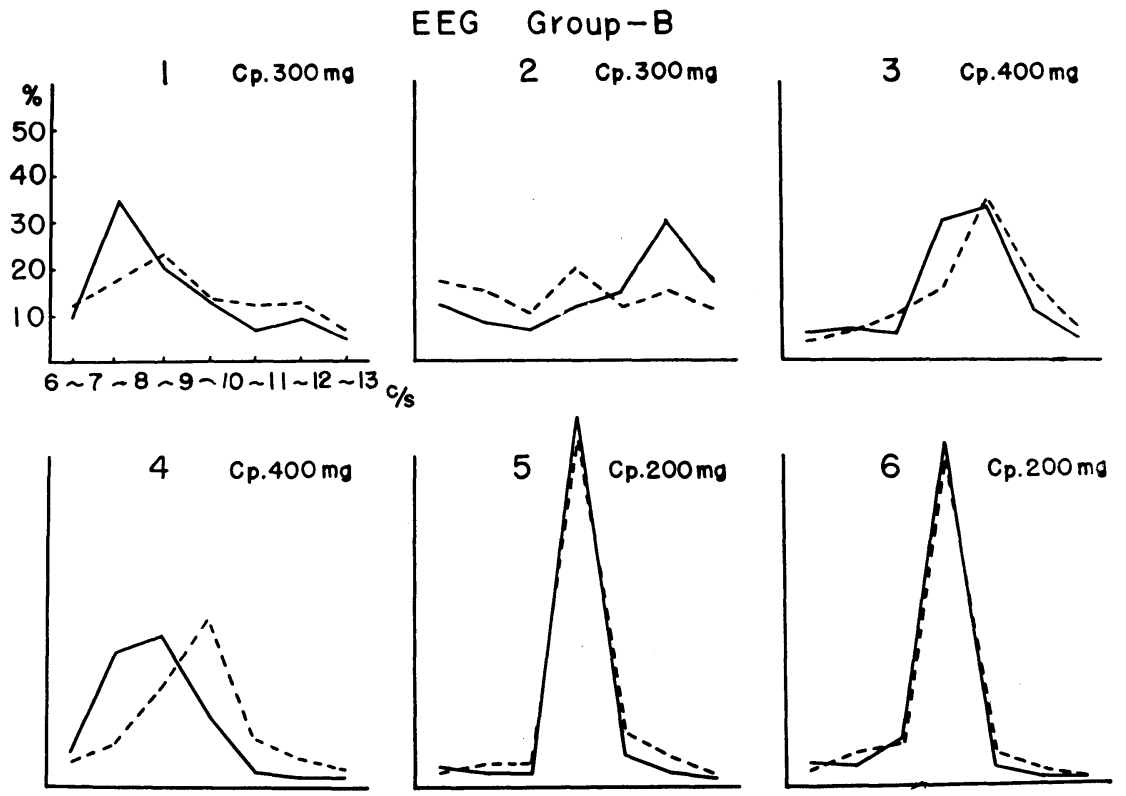

Fig. 2. Frequency graphs of EEG of six cases (Group B)

...... before medication

at the time with clinical improvement

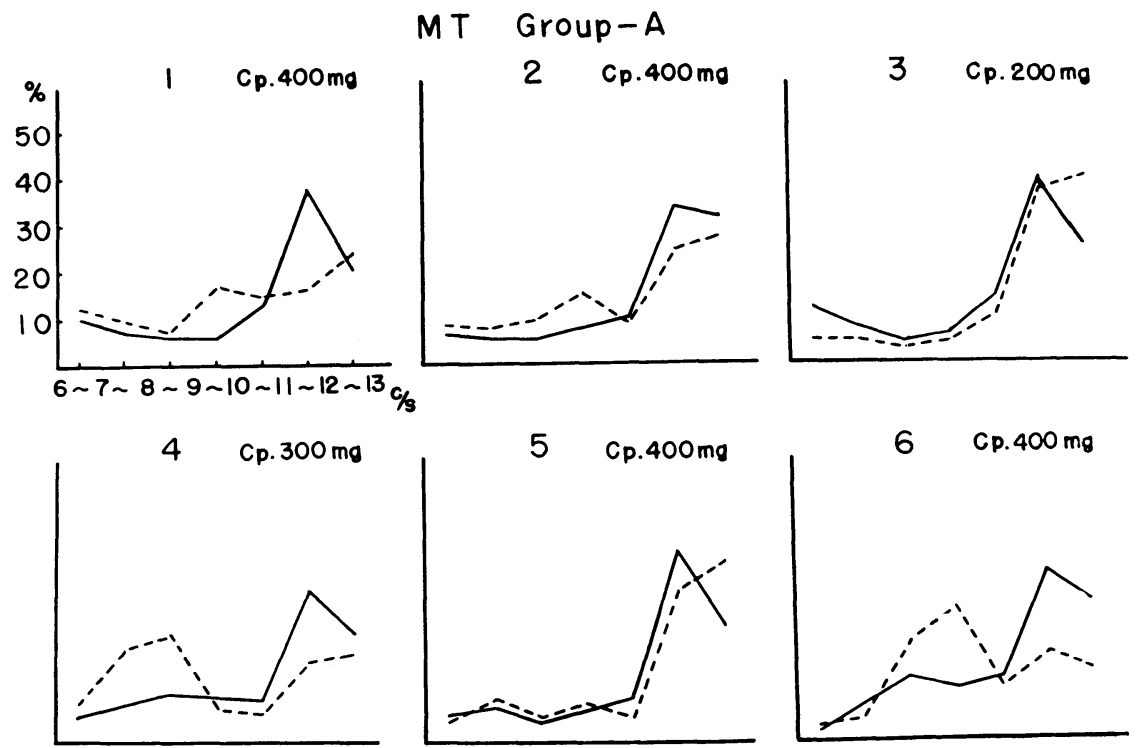

Fig. 3. Frequency graphs of MT of six cases (Group A)

....... before medication

at the time with clinical improvement 


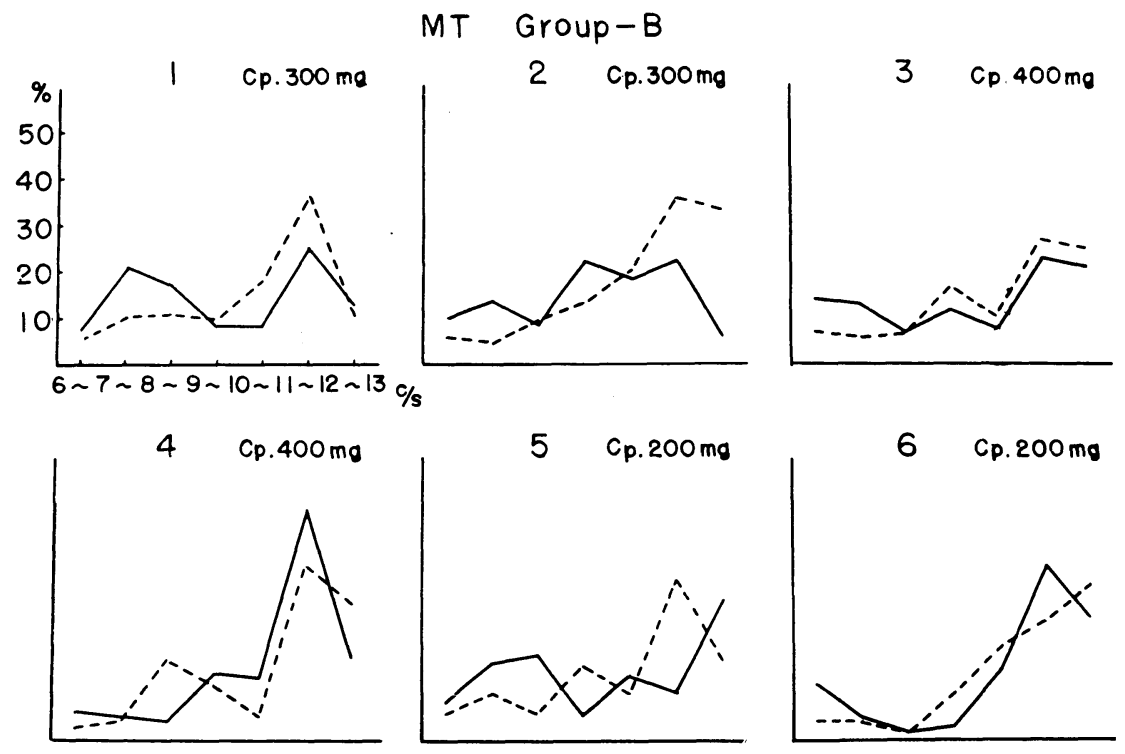

Fig. 4. Frequency graphs of MT of six cases (Group B)

$\cdots \cdots$ before medication

at the time without improvement,

randomly taken

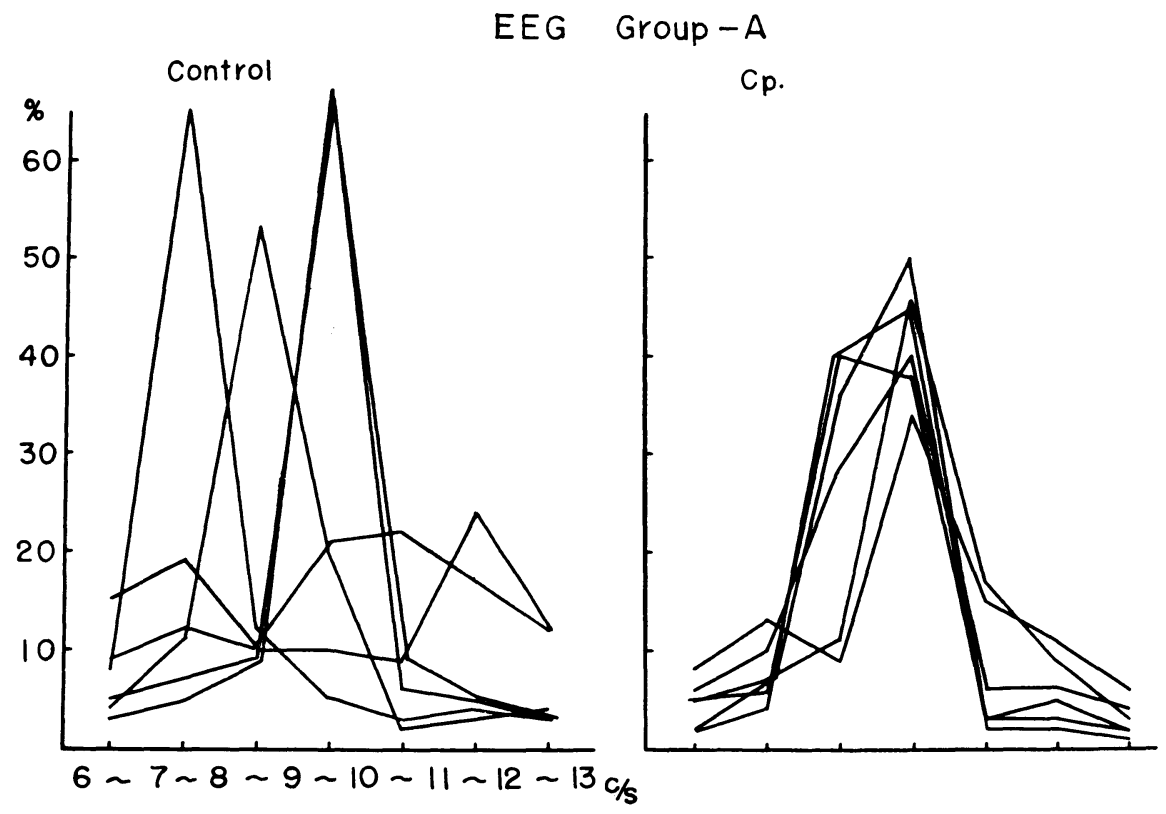

Fig. 5. The superimposed frequency graphs of EEG of Group A. left (control): before medication right $(\mathrm{Cp})$ : at the time with clinical improvement during chlorpromazine therapy 


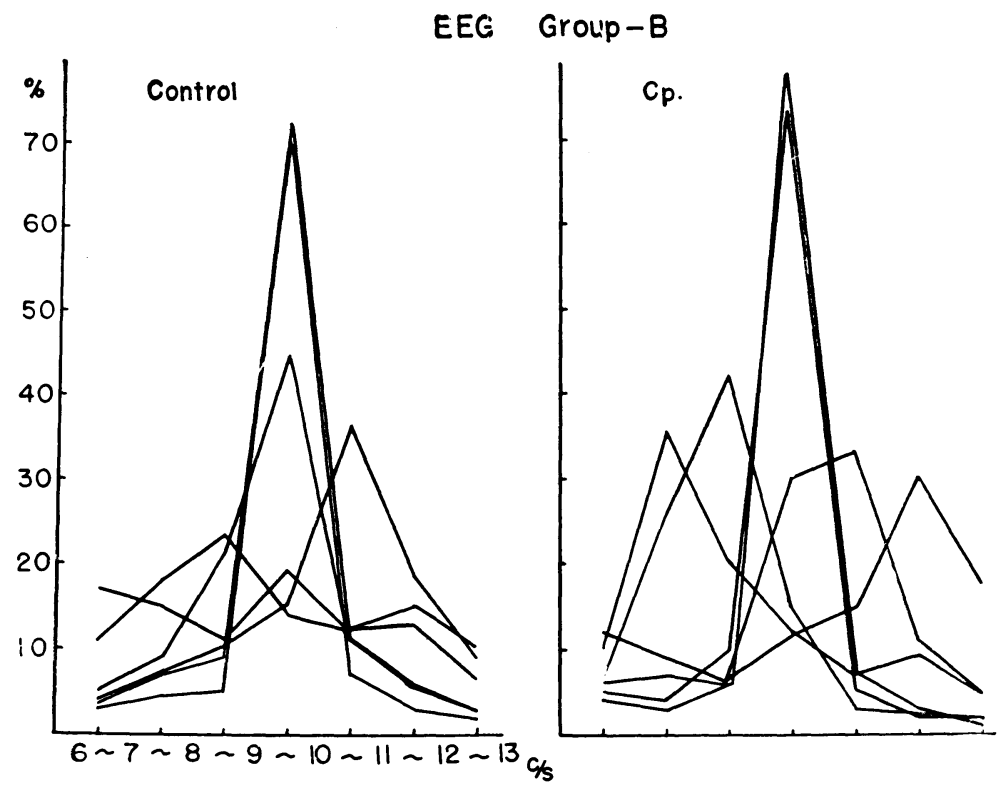

Fig. 6. The superimposed frequency graphs of EEG of Group B. left (control) : before medication right $(\mathrm{Cp})$ : at the time without clinical improvement, during chlorpromazine therapy

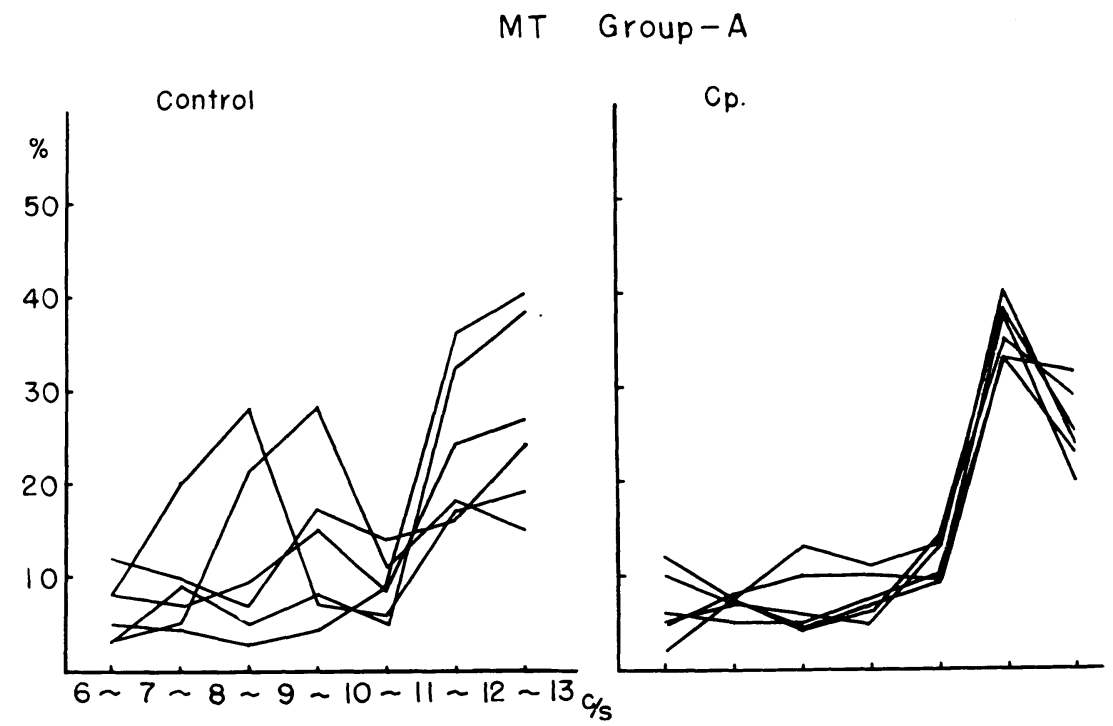

Fig. 7. The superimposed frequency graphs of MT of Group A. left (control): before medication right $(\mathrm{Cp})$ : at the time with clinical improvement, during chlorpromazine therapy 


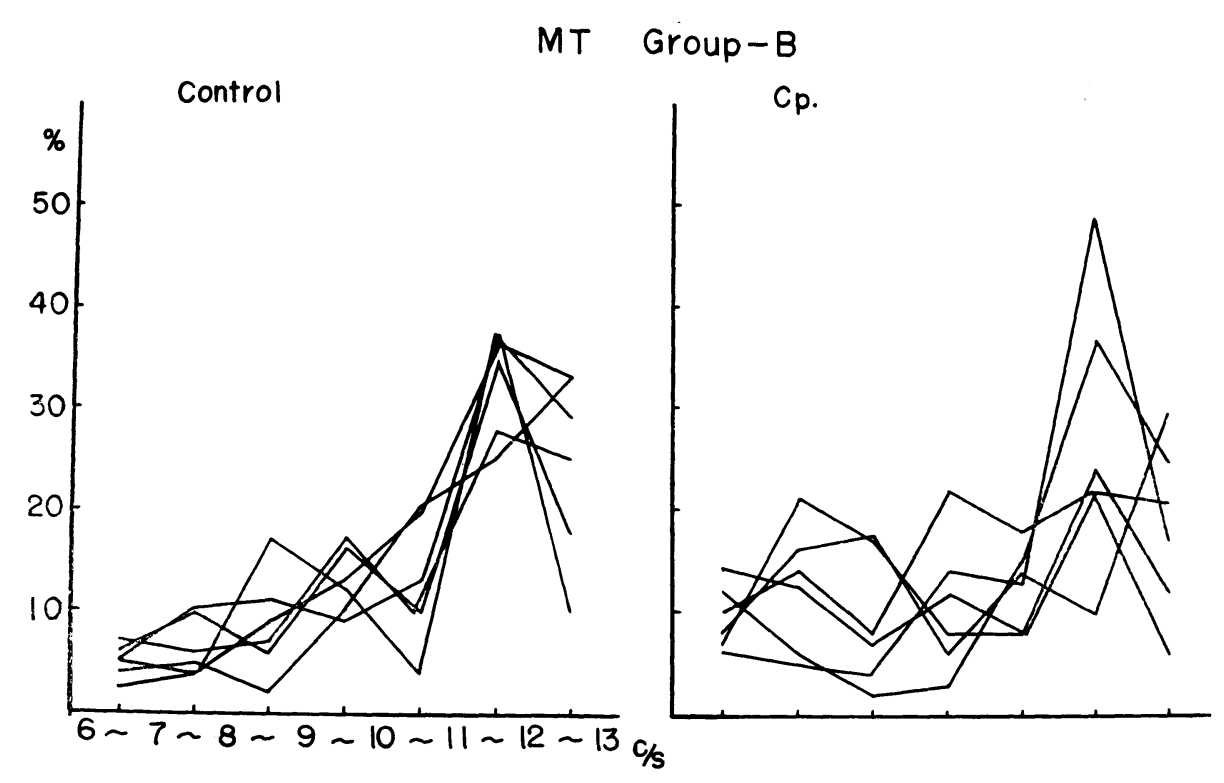

Fig. 8. The superimposed frequency graphs of MT of Group B.

left (control): before medication

right $(\mathrm{Cp})$ : at the time without clinical improvement

during $\mathrm{Cp}$ therapy

(3) Finally, the overall comparison of frequency curves of both EEG and MT during chlorpromazine treatment was made in Groups A and B. The difference is shown evidently in Figs. 5 and 6. EEG frequency curves in Group A have the normal pattern, with the peak at $9-10 \mathrm{c} / \mathrm{s}$ frequecy band. On the contrary, those of Group B show diverse changes. The same tendency was noticed in MT. MT frequency curves of Group A were well conformed and convergent with the peak at $11-12 \mathrm{c} / \mathrm{s}$ frequency band in all cases. On the contrary, those of Group B showed diversity of pattern (Figs. 7 and 8 ).

\section{DISCUSSION}

There are many reports ${ }^{1 / 6) 73912)}$ concerning the effects of phenothiazine derivatives on EEG. However only a few reports are concerned with the clinico-electroencephalographic correlations. Yamauchi ${ }^{12}$ did not find any significant relation between them. Mukasa et al. ${ }^{9}$ did not mention them. However, both reports confirmed that atypical frequency curves in the EEG tended to become typical after therapy. Fink ${ }^{2}$ reported that schizophrenic patients with high delta activity in EEG during electroshock therapy were found to be much improved (67\%), whereas only 30 per cent of the patients without such activity were so rated.

Igert et al. ${ }^{6)}$ pointed out that schizophrenic cases with normal EEG, showing the same electrical pattern despite intensive treatment, had the most unfavorable 
prognosis, and on the contrary, those with abnormal records showed a higher sensitivity to treatments from both the clinical and EEG point of view. In the present study, the same tendency was found, i.e., in the course of chlorpromazine therapy, a direct relationship has been observed between the degree of induced EEG and behavioral changes. Moreover, it seems to be one of the important factors for the behavioral improvement that the atypical EEG pattern shifts to normal. There are some reports ${ }^{34) 8}$ ) related to the effects of psychotropic drugs on MT. Yamauchi 12) pointed out the relation of behavioral improvement with the increase of alpha frequency band in MT.

Inanaga et al. ${ }^{5)}$ made serial observations of MT, before, during and after prolonged sleep treatment in depressive patients and found a close relation between the remarkable increase of alpha in MT and their clinical improvement after therapy.

Sugano et al. ${ }^{10)}$ observed the changes of MT under various experimental conditions and related the emotional changes to MT frequency curves. For example, it is possible to change MT by hypnotic suggestion, and when a subject is in a tranquil mind, the amplitude of MT decreases and the alpha frequency band increases.

Generally, in acute experiment, psychoactivators increased the amplitude and decreased the percentage of alpha band. On the contrary, psychohibitors decreased the amplitude and increased the percentage of alpha band.

In this study, the wave analyser was designed to divide the $6-13 \mathrm{c} / \mathrm{s}$ frequency range into seven frequency bands, in order to analyze the alpha frequency range in detail.

It was rather unexpected that the MT frequency curves in Group A were so well conformed and convergent in all cases. The conformity of MT frequency curves suggests the presence of a common denominator in the physiological basis of clinical improvement.

Summarizing the above mentioned results, it may be concluded that patients with favorable results had an atypical EEG and MT pattern before treatment, which could be altered to a normal pattern, and those with unfavorable results had a rather typical pattern before treatment, which could be changed to an abnormal pattern by chlorpromazine therapy.

\section{SUMMARY}

The relation between the physiological and clinical responses to chlorpromazine treatment was studied. EEG and MT were used as indicators of physiological responses.

1) The frequency curves of EEG before treatment showed 6 cases with alpha dominant pattern, 4 cases without dominant peak frequency and 2 cases of theta dominant pattern.

2) The frequency curves of EEG in six patients at the time of clinical improvement were evidently altered from those before treatment. The curves were almost in the normal range, with peak frequency at 9-10 c/s.

3) The frepuency curves of six patients without clinical improvement at any time during treatment were relatively unchanged, in comparison with curves before treatment. The curves were rather scattered. 
4) The frequency curves of MT before medication showed 10 cases dominating at faster frequency bands, one case with the peak frequency at $9-10 \mathrm{c} / \mathrm{s}$ and one case with the peak frequency at $8-9 \mathrm{c} / \mathrm{s}$.

5) In Group A, clinically improved by treatment, the frequency curves of MT in all subjects were well comformed and convergent with peak frequency at 11-12 c/s without exception. On the other hand, in clinically unimproved Group B, the curves were divergent.

6) It was found that there was a close relation between the clinical improvement and the pattern of frequency curves in EEG and MT.

\section{REFERENCES}

1) * Aкмиото, T. : Effects of barbiturate and tranquilizers on human electroencephalogram-automatic frequency analyses. Brain and Nerve, 12, 1001, 1960.

2) Fink, M. : Alteration of brain function in therapy. cited from Psychopharmacology Frontiers, 325, Little, Brown, 1959.

3) * Inanaga, K. : Minor Tremor and psychotherapeutic drugs. Shinkei-Kenkyu no Shinpo, 5, 663, 1961.

4) * InANAGA, K. : Minor tremor on the body surface. Igakusyuppan. Tokyo. 1960.

5) * Inanaga, K. and Ishibashi, A. : Serial observations of Minor Tremor, before, during and after prolonged sleep treatment. Brain and Nerve, 14, 439, 1962.

6) Igert, C. et LaILy, G.C. : Intérèt prognostique de 1'EEG au cours de 1'évolution des schizophrènes. EEG Clin. Neurophysiol., 14, 183, 1962.

7) Jörgensen, R.S. AND WulfF, M.H. : The effect of orally administered chlorpromazine on the electroencephalogram of man. EEG Clin. Neurophysiol., 10, 325, 1958.

8) Kurita, S. : The effect of chlorpromazine on the Minor Tremor. Kyushu J. Med. Sci. , 9, 236, 1958.

9) * Mukasa, H., Ichinara, T., Matsuoka, M. and Akivama, K. : Clinical and physiological studies on the effects of Fluphenazine, Brain and Nerve, 13, 431, 1961.

10) Sugano, H. and Inanaga, K. : Studies on minor tremor. Jap. J. Physiol., 10, 246, 1960.

11) * YaMAUCh, I. : A comparative study of Minor Tremors and EEGs in normal persons by frequency analysis. Brain and Nerve, 14, 169, 1962.

12) * YamaUCHI, I. : Study on the effects of perphenazine upon EEG and Minor Tremor in schizophrenics by means of automatic frequency analyser. Brain and Nerve, 13, 959, 1961 .

(Remark: *were written in Japanese) 